\title{
Nail involvement in psoriatic arthritis
}

\author{
Piotr Sobolewski, Irena Walecka, Klaudia Dopytalska
}

Department of Dermatology, Central Clinical Hospital of the Ministry of the Interior, Warsaw, Poland

\begin{abstract}
Nail psoriasis is considered a significant psychological and social problem causing functional impairment in affected patients. Nail changes hamper their daily and occupational activities and contribute to a worse quality of life. Almost $50 \%$ of patients with psoriasis vulgaris and up to $80 \%$ of patients with psoriatic arthritis are afflicted with nail lesions. The important correlation between psoriatic arthritis and nail changes is well established - the presence of the latter is a strong predictor of the development of arthritis. There is a broad spectrum of nail dystrophies associated with psoriasis, ranging from the common pitting, subungual hyperkeratosis and loosening of the nail plate to less frequent discolouration and splinter haemorrhages. Some of these symptoms are also observed in other nail diseases, and further diagnostics should be performed. The assessment tools NAPSI (Nail Psoriasis Severity Index), mNAPSI (Modified Nail Psoriasis Severity Index), and PNSS (Psoriasis Nail Severity Score) are most commonly used to grade the severity of nail involvement in psoriasis and enable the evaluation of therapy effectiveness. The treatment of nail psoriasis is a major clinical challenge. It should be adjusted to the extent of dermal, articular and ungual lesions. Systemic therapies of psoriasis, especially biological agents, are most likely to be effective in treating nail psoriasis. However, as their use is limited in scope and safety, topical therapy remains a mainstay, and the combination of corticosteroids and vitamin $D_{3}$ analogues is considered to be most helpful.
\end{abstract}

Key words: nail psoriasis, psoriatic arthritis, treatment of nail changes.

\section{Introduction}

Psoriasis is a chronic, systemic inflammatory disease involving skin, nails and joints. Most patients with psoriasis have nail involvement. It is considered as an important psychological and social problem. Nail changes are not only a cosmetic defect, but also may lead to many difficulties in daily activities including work and impairment in quality of life. Nail involvement in psoriasis is also the cause of severe pain, anxiety and depression [1-3]. Treatment of nail psoriasis may be highly challenging, because of its resistance to therapy.

A significant correlation between nail psoriasis and psoriatic arthritis is established. Patients with skin psoriasis have a rate of nail disease of approximately $40 \%$, while the rate of nail involvement in psoriatic arthritis is up to $80 \%[1,4-6]$. There is strong evidence that nail psoriasis is a predictor of joint disease and may occur even a few years earlier before arthritis symptoms [7].

\section{Nail structure}

The nail unit is comprised of the matrix, the nail plate, the nail bed and the nail folds. The matrix is responsible for nail growth. It can be divided into proximal and distal parts. Nails grow toward the distal part over the nail bed. The distal part of the nail plate is connected to the nail bed by the hyponychium. Within the proximal part of the nail plate, there are white semi-circular areas known as lunulae. The nail is surrounded by the lateral nail folds and the proximal nail fold (PNF) $[1,8,9]$.

The main function of the nail unit is to protect the fingertips from traumatic injuries. In addition, nails enhance precise movement ability of the fingers, which is

Address for correspondence:

Piotr Sobolewski, Department of Dermatology, Central Clinical Hospital of the Ministry of the Interior, Woloska 137, 02-507 Warsaw,

Poland, e-mail: piotr.sobolewski@cskmswia.pl

Submitted: 10.04.2017; Accepted: 1.06.2017 
necessary to manipulate small objects [1]. Patients with nail diseases may have issues with precise movement and often feel discomfort and pain [8].

\section{Nail lesions in psoriasis}

Clinically, nail psoriasis has many manifestations, which depend on the location of the inflammatory process. Nail matrix involvement causes nail pitting, Beau's lines, onychomadesis, trachyonychia, nail dystrophy and leukonychia, whereas nail bed psoriasis leads to onycholysis, oil drop patches, subungual hyperkeratosis and splinter haemorrhages $[2,10]$.

The most common nail sign in psoriasis is pitting, which affects approximately $68 \%$ of patients with psoriasis and nail changes. It is also the most common symptom in psoriatic arthritis [1]. Pits are superficial depressions within the nail plate and are associated with inflammation of the proximal nail matrix. Nail pitting correlates with activity of psoriasis - the length of pits suggests time of the disease, but the length of the healthy nail (between pits) correlates with time free from psoriasis [1, 11]. Pitting also may appear in cases of other diseases such as eczema, alopecia areata, and lichen planus, but the pits are usually deeper in psoriasis [12] (Fig. 1).

Another typical manifestation of nail psoriasis is onycholysis-detachment of the nail plate from the nail bed.

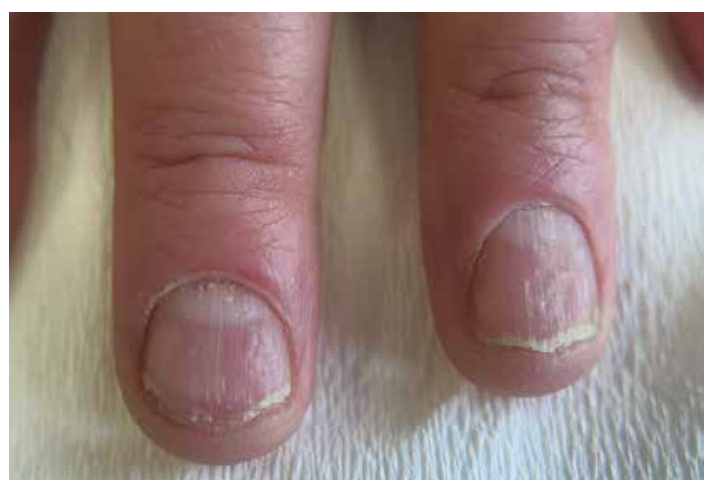

Fig. 1. Nail psoriasis. Pitting.

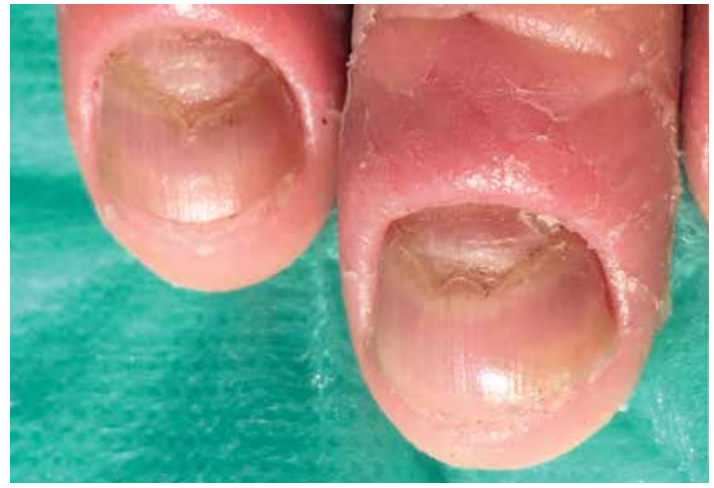

Fig. 2. Onychomadesis in nail psoriasis.
The separation usually starts at the tip and progresses to the proximal part of the nail. The newly formed space is a potential place for entry and colonisation for various microorganisms and increases the risk of infection [1, 2]. In severe cases of nail psoriasis, the inflammation may lead to complete shedding of the nail (onychomadesis) (Fig. 2). Onycholysis can also be caused be nail trauma, fungal infection or thyroid gland diseases [8] (Fig. 3).

Subungual hyperkeratosis is a result of the deposition and collection of cells which have not undergone desquamation. It is associated with inflammation involving the nail bed and hyponychium. Subungual hyperkeratosis is a common lesion in nail psoriasis, especially in the toenails, which leads to significant cosmetic defects and issues with daily activities [1, 2, 11] (Fig. 4).

Inflammation of the matrix and proximal nail folds in psoriasis can cause transverse grooves and ridges within the nail plate, known as Beau's lines [2, 13]. Beau's lines usually occur on every fingernail or toenail. These lines are also a symptom of nail trauma or low temperature exposure in patients with Raynaud's disease [12]. As a result of long-term matrix involvement the nail plate can assume a crumbly appearance (crumbling) with rough linear ridges (trachyonychia) [1, 2].

Typical symptoms of nail psoriasis are discolourations. Oil drops or salmon patches are translucent yellow-red discoloration in the nail bed, which are pathognomonic sign of psoriasis $[11,14,15]$. White spots on the nail, known as leukonychia, are induced by parakeratosis within the nail plate as an effect of psoriasis in the mid-matrix (Fig. 5).

Splinter haemorrhages are tiny, linear, "splinter" shaped clots. These haemorrhages are caused by the rupture of delicate capillaries between the nail bed and the nail plate. The most common etiology is trauma, but splinter haemorrhages are also associated with vasculitis or bacterial endocarditis [1, 11] (Fig. 6).

The nail changes in psoriasis can occur in both the fingernails and toenails. In psoriatic arthritis the most common manifestations are pitting and sublingual hyperkeratosis [16, 17]. Similar symptoms are also associated with many other dermatological diseases, so

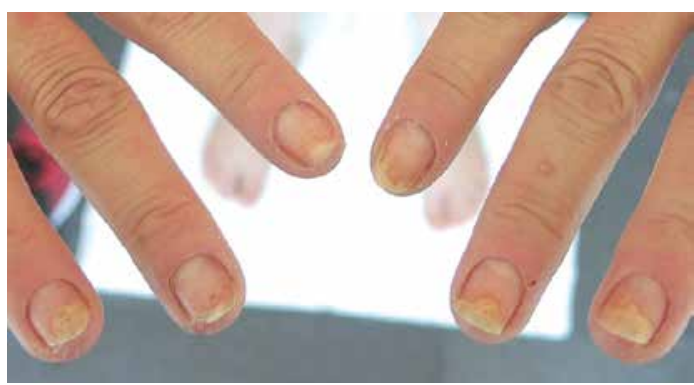

Fig. 3. Onycholysis in nail psoriasis. 


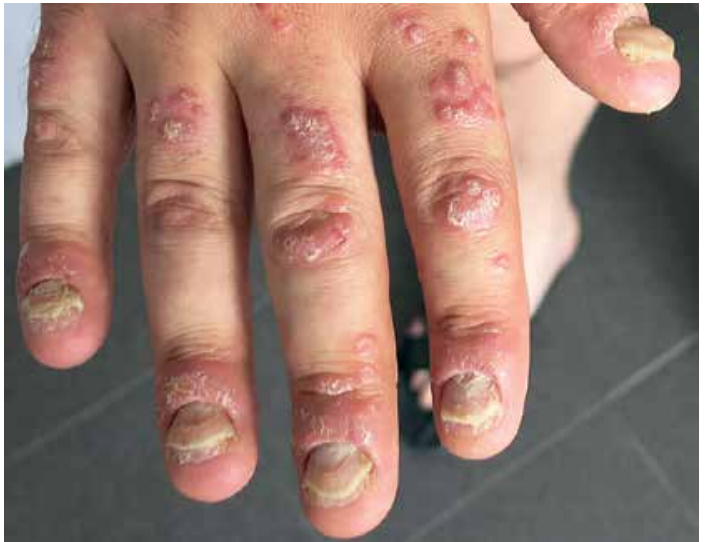

Fig. 4. Nail psoriasis. Subungual hyperkeratosis.

a differential diagnosis may be necessary in some cases (Fig. 6, 7).

\section{Diagnosis and evaluation of nail psoriasis progression}

Nail psoriasis is so characteristic that in the vast majority of cases it can be diagnosed only by the clinical features. Nevertheless, some other nail diseases should be differentiated, because only the oil-drop sign is considered to be pathognomonic of psoriatic nails.

In the differential diagnosis particularly onychomycosis should be taken into consideration. The fungal lesions of the nails can resemble those in psoriasis, but both diseases can be concomitant. The anomalous, dystrophic nail plate in psoriasis provides a great site for dermatophytes, Mucor and Candida [2]. A mycological examination is recommended in both cases - every onychomycosis should be properly treated. Additionally, a negative result of this test is crucial in diagnosing a rare type of psoriasis - isolated nail psoriasis. This variety frequently precedes full-blown psoriasis.

Isolated onycholytic changes of the distal or marginal parts of the nail plate can bring some problems in

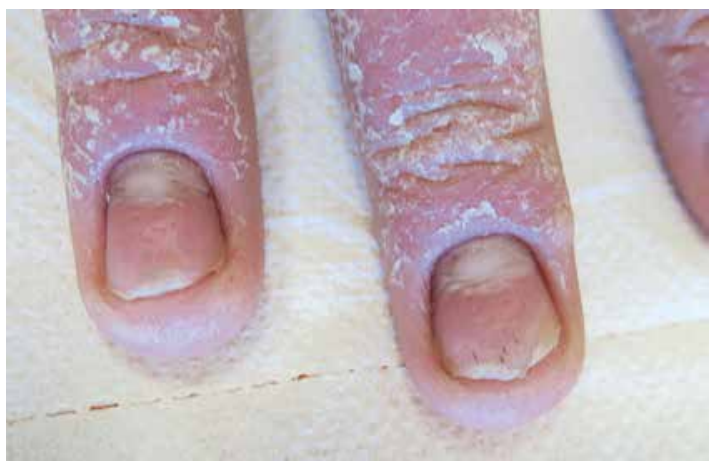

Fig. 6. Nail psoriasis. Splinter hemorrhages.

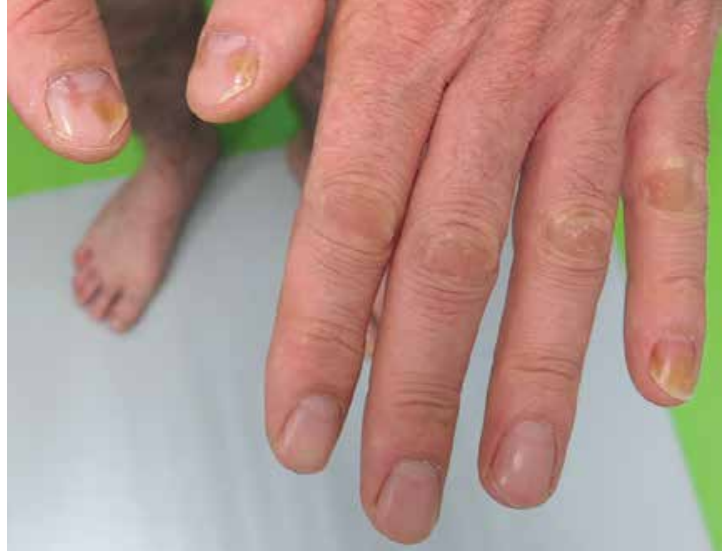

Fig. 5. Oil drop sign in nail psoriasis.

differentiation. In such cases lichen planus should be excluded. Only long-term observation over several months or years can lead to a proper diagnosis.

Several scales have been developed in order to evaluate the degree of psoriatic progression in the nails, including the Nail Psoriasis Severity Index (NAPSI), the Modified Nail Psoriasis Severity Index (mNAPSI), and the Psoriasis Nail Severity Score (PNSS). In the PNSS scale one point is given for the presence of each change from the following list: pitting, subungual hyperkeratosis, onycholysis and advanced deformation of both nail ends. The maximum score is 4 for a nail. The NAPSI scale grades the psoriatic changes of the nails differently - the nail is divided with imaginary horizontal and longitudinal lines into quadrants. Each nail is given a score for bad nail lesions ( $0-4$ points) and nail matrices ( $0-4$ points) depending on the presence of any of the features in that quadrant. Nail matrix psoriasis is characterized by nail plate changes of pitting, leukonychia, red spots in the lunula and nail plate crumbling. Nail bed psoriasis shows onycholysis,

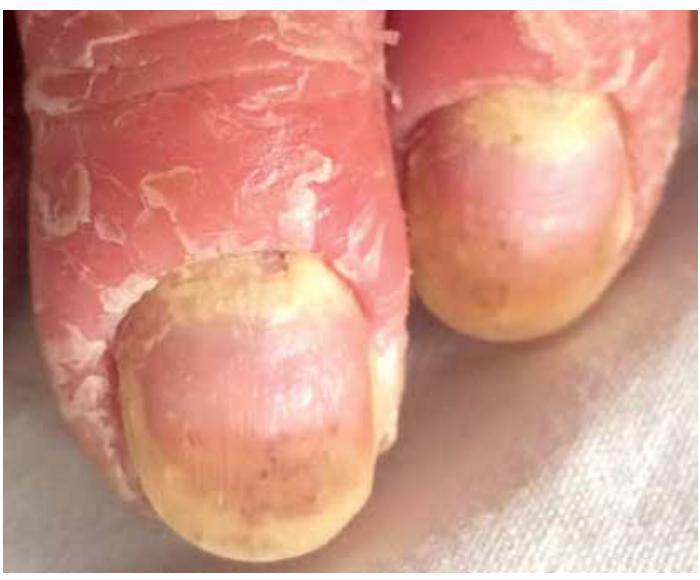

Fig. 7. Advanced nail changes in severe psoriasis - nail pitting, onycholysis, subungual hyperkeratosis and splinter haemorrhages. 
oil-drop dyschromia, splinter haemorrhages and subungual hyperkeratosis. Thus, for each nail the NAPSI score can amount to 8 points. As an alternative, a target nail can be selected and graded by means of evaluation for the presence of all 8 criteria in each quadrant of the nail with a total sum of 32 points. The modified Nail Psoriasis Severity Index (mNAPSI) scale also takes into account all 8 psoriatic changes of the nail without dividing it into parts, but the oil-drop syndrome and onycholysis are combined together in one criterion. Additionally, in nail evaluation using mNAPSI scale lesions, oil-drop dyschromia/onycholysis and dystrophy are graded from 1 to 3 in terms of severity. The presence of others are scored as 1 point. The maximum score is 13 points for each nail. The mNAPSI scale is considered to be an objective, numeric, reproducible grading system of nail psoriasis with a relatively simple algorithm of evaluation [18].

\section{The correlation between different manifestations of psoriasis}

Psoriatic arthritis (PsA) develops in $7-26 \%$ of those with psoriasis vulgaris $[7,19]$. The presence of skin lesions precedes articular symptoms typical of PsA in $75-80 \%$ of patients with a mean estimated delay of 10 years [20]. However, nail changes are observed in $40 \%$ of psoriasis patients and up to $80 \%$ of those with PsA [21]. This fact became the basis for numerous studies exploring the relationship of nail psoriasis and inflammatory changes in joints. The anatomical connection between the nail matrix and the enthesis of the distal interphalangeal (DIP) joint extensor was proved. The whole structure is called a joint-entheseal-nail apparatus [20]. As the DIP joints are the most often affected in PSA, involvement of this complex can be a possible explanation of considerably higher concomitance of nail changes in those patients. It was confirmed in the studies by imaging early or advanced inflammation in DIP joints and entheses by ultrasound and high-resolution MRI [22-24]. The entheses, like the skin, are exposed to both shear and compressive forces, which causes lesion spreading according to the Koebner phenomenon - mechanical stress and microdamage in DIP joints can result in psoriatic nails.

Some reports indicate that nail psoriasis is associated with a few predisposing factors. It was proven that nail changes are more frequently observed in patients with a long history of psoriasis vulgaris, early onset of psoriasis, higher score of Psoriasis Area and Severity Index (PASI) and with concomitant PSA [25].

\section{Treatment of nail psoriasis}

Treatment of nail psoriasis is a clinical challenge. The methods of treatment should be adjusted to the ex- tent of skin diseases, psoriatic arthritis, severity of nail changes and impairment of the quality of life. Topical products, procedural intervention, and systemic and biological agents are possible options for treatment. There are many impediments to therapy including poor penetration of topical products, adverse effects of systemic and biologic treatment, monitoring of improvements and patients' attitude [4].

Topical products are highly recommended in cases of patients with psoriasis limited to the nails, with minimal or without skin disease, and with no evidence of arthritis. Strong topical corticosteroids alone or in combination with calcipotriol are the best therapeutic option in these cases [2, 4, 5, 26, 27]. Intralesional injection of corticosteroids may be effective therapy but implies adverse effects such as pain of injection, skin atrophy, depigmentation, secondary infection and subungual haemorrhage. Triamcinolone acetonide $0.1-0.2 \mathrm{ml}$ of $5-10 \mathrm{mg} / \mathrm{ml}$ is most frequently used in injection. Nerve blockers or topical anaesthetics are necessary during procedures to reduce the pain [4].

Systemic therapy is highly effective in nail psoriasis and should be used when topical therapy has failed in patients with skin disease or psoriatic arthritis. Methotrexate and cyclosporine can improve the nail lesions. In a comparison of these two agents, methotrexate is more effective in treating nail matrix changes, while cyclosporine is more effective in nail bed scores [28]. Acitretin can reduce the NAPSI score, but may increase onycholysis and pitting [2, 4, 29, 30].

Biological therapy is a great achievement in nail psoriasis treatment. The recommendations for therapy, in ranked order from the highest to lowest, are adalimum$a b$, etanercept, ustekinumab, infliximab, apremilast and golimumab [4, 31-36].

Psoriatic nails are often secondarily infected with dermatophytes, which may complicate therapy. Therefore, patients with nail psoriasis should have fungal nail infection excluded [4].

Clinical data are limited in nail psoriasis and evaluation of treatment is not standardized. It is necessary to adjust the method of therapy to the extent of skin diseases, psoriatic arthritis, severity of nail changes and impairment of quality of life. Even when skin psoriasis and psoriasis arthritis are controlled, nail lesions can be still present.

The authors declare no conflict of interest.

\section{References}

1. Jiaravuthisan MM, Sasseville D, Vender RB, et al. Psoriasis of the nail: anathomy, pathology, clinical presentation, and a re- 
view of the literature on therapy. J Am Acad Dermatol 2007; 57: 1-27.

2. Wolska H. Nail psoriasis. Przegl Dermatol 2010; 97: 243-253.

3. de Jong EM, Seegers BA, Gulinck MK, et al. Psoriasis of the nails associated with disability in a large number of patients: results of a recent interview with 1728 patients. Dermatology 1996; 193: 300-303.

4. Crowley J, Weinberg J, Wu J, et al. Treatment of nail psoriasis: best practice recommendations from the Medical Board of the National Psoriasis Foundation. JAMA Dermatol 2015 151: 87-94.

5. Reich K. Approach to managing patients with nail psoriasis J Eur Acad Dermatol Venereol 2009; 23 Suppl 1: 15-21.

6. Armesto S, Esteve A, Coto-Segura P, et al. Nail psoriasis in individuals with psoriasis vulgaris:a study of 661 patients. Actas Dermosifiliogr 2011; 102: 365-372.

7. Raposo I, Torres T. Nail psoriasis as a predictor of the development of psoriatic arthritis. Actas Dermosifiliogr 2015; 106 : 452-457.

8. Braun-Falco O, Plewig G, Wolff HH. Dermatology 2000; 10731078.

9. Achten G, Parent D. The normal and pathologic nail. Int J Dermatol 1983; 22: 556-565.

10. Grover C, Reddy BS, Uma Chaturvedi K. Diagnosis of nail psoriasis: importance of biopsy and histopathology. Br J Dermato 2005; 153: 1153-1158.

11. Zaias N. The nail in health and disease. 2nd ed. Appleton \& Lange, Norwalk 1990.

12. Fawcett RS, Linford S, Stulberg DL. Nail abnormalities: clues to systemic disease. Am Fam Physician 2004; 69: 1417-1424.

13. Scher RK, Daniel CR. Nails: therapy, diagnosis, surgery. W.B. Saunders Company, Philadelphia 2003.

14. Schons KR, Knob CF, Murussi N, et al. Nail psoriasis: a review of the literature. An Bras Dermatol 2014; 89: 312-317.

15. Tan ES, Chong WS, Tey HL. Nail psoriasis: a review. Am J Clin Dermatol 2012; 13: 375-388.

16. Salomon J, Szepietowski JC, Proniewicz A. Psoriatic nails: a prospective clinical study. J Cutan Med Surg 2003; 7: 317-321.

17. Lavaroni G, Kokelj F, Pauluzzi P, et al. The nail in psoriatic arthritis. Acta Derm Venereol Suppl (Stockh) 1994; 186: 113.

18. Sandre MK, Rohekar S. Psoriatic arthritis and nail changes: exploring the relationship. Semin Arthritis Rheum 2014; 44: 162-169.

19. Prey S, Paul C, Bronsard V, et al. Assessment of risk of psoriatic arthritis in patients with plaque psoriasis: a systematic review of the literature. J Eur Acad Dermatol Venereol 2010; 24 Suppl 2: 31-5.

20. Langenbruch A, Radtke MA, Krensel M, et al. Nail involvement as a predictor of concomitant psoriatic arthritis in patients with psoriasis. Br J Dermatol 2014; 171: 1123-1128.

21. Raposo I, Torres T. Nail psoriasis as a predictor of the development of psoriatic arthritis. Actas Dermosifiliogr 2015; 106: 452-457.

22. Tan AL, Benjamin M, Toumi H, et al. The relationship between the extensor tendon enthesis and the nail in distal inter- phalangeal joint disease in psoriatic arthritis - a high-resolution MRI and histological study. Rheumatology (Oxford) 2007; 46: 253-256.

23. Aydin SZ, Castillo-Gallego C, Ash ZR, et al. Ultrasonographic assessment of nail in psoriatic disease shows a link between onychopathy and distal interphalangeal joint extensor tendon enthesopathy. Dermatology 2012; 225: 231-235.

24. Sandre MK, Rohekar S, Guenther L. Psoriatic Nail changes are associated with clinical outcomes in psoriatic arthritis. J Cutan Med Surg 2015; 19: 367-376.

25. Schons KR, Beber AA, Beck Mde O, et al. Nail involvement in adult patients with plaque-type psoriasis: prevalence and clinical features. Bras Dermatol 2015; 90: 314-319.

26. Rigopoulos D, Gregoriou S, Daniel CR III, et al. Treatment of nail psoriasis with a two-compound formulation of calcipotriol plus betamethasone dipropionate ointment. Dermatology 2009; 218: 338-341.

27. Rigopoulos D, loannides D, Prastitis N, et al. Nail psoriasis: a combined treatment using calcipotriol cream and clobetasol propionate cream. Acta Derm Venereol 2002; 82: 140.

28. Gümüşel M, OzdemirM, Mevlitoğlu I, et al. Evaluation of the efficacy of methotrexate and cyclosporine therapies on psoriatic nails: a one-blind, randomized study. J Eur Acad Dermatol Venereol 2011; 25: 1080-1084.

29. Brazzelli V, Martinoli S, Prestinari F, et al. An impressive therapeutic result of nail psoriasis to acitretin. J Eur Acad Dermatol Venereol 2004; 18: 229-230.

30. Baran R. Retinoids and the nails. J Dermatol Treat 1990; 1 : 151-154.

31. Ortonne JP, Paul C, Berardesca E, et al. A 24-week randomized clinical trial investigating the efficacy and safety of two doses of etanercept in nail psoriasis. Br J Dermatol 2013; 168: 1080-1087.

32. Kavanaugh A, McInnes I, Mease P, et al. Golimumab, a new human tumor necrosis factor-antibody, administered every four weeks as a subcutaneous injection in psoriatic arthritis: twenty-four-week efficacy and safety results of a randomized, placebo-controlled study. Arthritis Rheum 2009; 60: 976-986.

33. Reich K, Ortonne JP, Kerkmann U, et al. Skin and nail responses after 1 year of infliximab therapy in patients with moderate-to-severe psoriasis: a retrospective analysis of the EXPRESS Trial. Dermatology 2010; 221: 172-178.

34. Igarashi $A$, Kato $T$, Kato $M$, et al. Japanese Ustekinumab Study Group. Efficacy and safety of ustekinumab in Japanese patients with moderate-to-severe plaque-type psoriasis: longterm results from a phase $2 / 3$ clinical trial. J Dermatol 2012; 39: 242-252.

35. Leonardi C, Langley RG, Papp K, et al. Adalimumab for treatment of moderate to severe chronic plaque psoriasis of the hands and feet: efficacy and safety results from REACH, a randomized, placebo-controlled, double-blind trial. Arch Dermatol 2011; 147: 429-436.

36. Rich P, Griffiths CE, Reich K, et al. Baseline nail disease in patients with moderate to severe psoriasis and response to treatment with infliximab during 1 year. J Am Acad Dermatol 2008; 58: 224-231. 